\title{
Computer Vision system to aid drivers of vehicles through vertical signaling traffic
}

\author{
Elizângela de Souza Rebouças \\ Federal Institute of Technology of Ceará \\ Av. Treze de Maio, 2081 \\ Benfica, Zip-code: 60040-531
}

\author{
Samuel Luz Gomes \\ PPGER - Federal Institute of Technology of Ceará \\ Av. Treze de Maio, 2081 \\ Benfica, Zip-code: 60040-531
}

\author{
Pedro Pedrosa Rebouças Filho \\ PPGER - Federal Institute of Technology of Ceará \\ Av. Treze de Maio, 2081 \\ Benfica, Zip-code: 60040-531
}

\begin{abstract}
The constant increase in the number of vehicle, especially in large urban centers, a growing number of accidents caused by the invasion of neighboring groups of drivers, either by inattention, drowsiness, among others. To mitigate the impact of this problem, this paper proposes the Aid System the Driver Vehicle (ASDV), which is based on a system uses computer vision techniques and Digital Image Processing to detect the tracks of markings and through these identify the behavior that the car driver must have to continue in its tracks of movement on the road. Tests are performed on Unix platform and the Android operating system, obtaining the speed $50 \mathrm{fps}$ and $20 \mathrm{fps}$, respectively. The tests show satisfactory results, achieving $100 \%$ accuracy when the tracks are detected. Therefore, we can conclude that the system is promising and shows potential to be used in real applications.
\end{abstract}

\section{General Terms:}

Vertical traffic signs, aid to the car driver, Computer Vision

\section{Keywords:}

Vertical traffic signs, traffic lines, Digital Image Processing, Computer Vision System, Aid to the car driver.

\section{INTRODUCTION}

With the constant increase of the vehicle fleet, especially in large urban centers, problems arise more and more difficult to be solved which thus require the development of improved techniques for the control of traffic. Currently, only the state of Cear have a fleet of about two million vehicles and the state of So Paulo there are more than twenty-four million.

It is known that all the traffic signs serves to regulate, warn, advise, inform and monitor the movement of vehicles and pedestrians on the land routes. Among these there are the road markings are painted on the road bed being the most common and known, among others, the range of the pedestrian and the continuous and dashed lines. The latter serve to delimit the space where vehicles may or may not move, and indicates the overtaking places and show the direction of the track. However, whether by recklessness or inattention, many of these signs are disregarded or ignored, causing often accidents on the roads. According to the DNIT (National Department of Transport Infrastructure) in 2011 were recorded 188,925 accidents, of which 24,933 were for "off the track." These data demonstrate the great need to develop technologies that help drivers reducing thus the number of accidents [2].

Because of the limitations of these mechanisms, there is a demand for enforcement strategies, control, management, and prevention of more intelligent and efficient traffic accidents and the computer vision systems have been considered as a viable alternative for the development of solutions that can meet the this demand [10, $8,9,14,5,11]$. Simultaneously, the search fields in this area are growing every day with efficient solutions to various problems. In this scenario, develop this type of system has been a priority in many countries, since several studies have sought to use computer vision techniques to increase the efficiency of traffic monitoring mechanisms [4].

The US Department of Transportation estimates that an investment of US 1.2 billion in ITS technology (Intelligent Transportation Systems) would generate a US 30.2 billion return in about 20 years. Similarly, since 2004 Japan invests US 700 million in these technologies, while South Korea plans to invest US 3.2 billion between 2008 and 2020 [5].

And is aiming to contribute to improvement in traffic control in Brazil this work was developed. The name of the application is developed System of Aid to the Vehicle Driver (ARCS), whose basic operating principle of the detection of vertical signaling tracks to the front of the vehicle and from these lines, assist the driver in the vehicle driving. This system is designed to minimize accidents by addressing its root causes, such as, distraction and drowsiness. To accomplish this, driving patterns are detected and signals are sent to the driver by audible, visual and/or vibratory signals. 


\section{EXPERIMENTAL PROCEDURES}

The Computer Vision system developed in this application follows the same methodology of a typical computer vision system, and consist of the steps of image acquisition, preprocessing, segmentation, extraction of attributes, identification of previously established standards and, finally, is generated a report with analysis and information obtained, as shown in Figure 1 [6, 13, 12, 3].

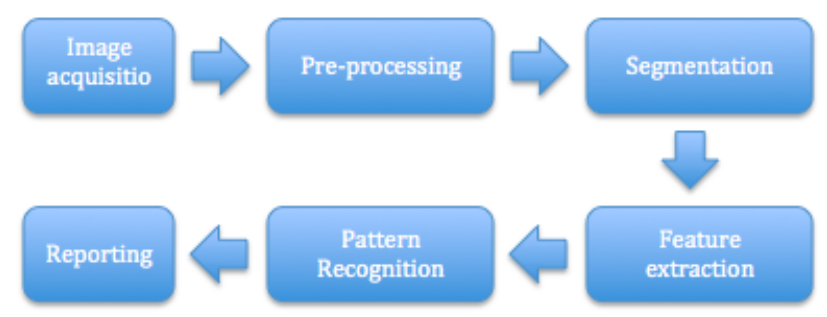

Fig. 1. SVC steps developed for detection and recognition of regulatory speed road signs [6].

According the methodology presented in Figure 1, the operation of ARCS flowchart shown in Figure 3, with an image acquisition step shown in green, the pre-processing step shown in blue with 3 Digital Images Processing methods (PDI), the segmentation in red, extraction of attributes in orange and the analysis presented in lilac. An example of each step is shown beside their respective step in the flowchart and detailed below.

Considering the steps in the Figure, it is necessary to detail the methods used in the proposed ARCS system 2

\subsection{Image Acquisition}

The image acquisition step is obtained by a camera of $720 \times 480$ pixels, wherein the positioning thereof may influence the results due be necessary to track the images as close to the vehicle. The system was developed to install this camera in the rearview mirror of the vehicle used by the driver.

\subsection{Preprocessing}

The pre-processing stage is characterized by the use of classical functions of PDI in order to make the most appropriate image for the developed application. In this system we used three techniques in sequence: clipping of the region of interest, conversion to grayscale and Canny operator.

The input image has many objects and information, however the region of interest for analysis is always the same, the bottom of the image just ahead of the motor vehicle. This zone can be configured in the system, and the area used is shown in red in the image of this step in Figure 2

The image is initially acquired in RGB (Red, Green, Blue) is a format used for streaming color images and is based on trichromatic color vision theory. In RGB format, each pixel is represented by three values in the case of an 8-bit image, values between 0 and 255 , the combination of these values represents a pixel color, and the image comprises three channels, each channel is as a matrix containing values for red, green and blue for each pixel, the combination of these channels generates vectors which represent the color of each pixel from black $(0,0,0)$ to white $(255,255,255)$. For converting RGB to Grayscale (Gray) is calculated average RGB vector of each pixel. The value found is the representation of the pixel value in gray. This conversion causes loss of information. The red $(255,0,0)$ and green $(0,255,0)$ are represented by the same value when converted to grayscale, this example explains the loss of information in the conversion process, however, this step becomes is important in computational point of view, since it reduces by onethird the amount of data to be processed.

After the picture is grayscale, the Canny operator is applied to detection of these edges in the image, which can be achieved by an approach for optimal detector step edges is the first derivative of a Gaussian, which in one dimension can be given by [6]:

$$
d=\left(-\frac{x}{\sigma^{2}}\right) e^{-\frac{x^{2}}{2 \sigma^{2}}}
$$

where $\sigma^{2}$ is the variance of the data and $x$ the input data.

\subsection{Segmentation}

Segmentation is the process of separating the various regions that make up an image, according to their properties. The segmentation technique to be used varies with the system application. The segmentation process is of fundamental importance in any Artificial Vision system, so that system performance is essentially dependent on the segmentation process performance. A segmentation performed properly on a computer vision system is a substantially important step to work correctly [1].

In the proposed system, the object of interest are the lines of road signs, the tracks that define the region where each car must be moved in the motor vehicle lanes. For this, the edges found by the Canny operator is analyzed at this stage.

Because the camera is installed in the vehicle rearview mirror, then we can assume that it is in the middle of this vehicle. Therefore the image taken by the camera shows the distance between the car and the tracks left and right when we consider an imaginary line in the input image center must have the same distance to the left lane and the right lane, as this line is the rear of the vehicle where the camera is installed.

In order to detect the signal of the left tracks, some lines are plotted in the horizontal direction based on the center imaginary line towards the left image, as shown in Figure 3(a), wherein the imaginary line is in red and the lines try left of the track are in green. When you find the first edge, each line that stores the position found for further analysis.

The same is done for the right lane detection, in which the lines run from the center toward the right in the horizontal direction and to detect the first edge, this position is stored. In Figure 3(a) the lines seeking the right lane are shown in blue. The number of rows and the spacing between them may be configured in the developed application, as shown in variable spacing (dl) of Figure $3(\mathrm{a})$

\subsection{Calculation of attributes}

The coordinates of the lines found in the segmentation step is used as input in this step, where only horizontal lines are the left and right tracks are analyzed. When three consecutive lines are the vertical signs tracks, then calculates the midpoint between these coordinates. Figure 3(b) shows an example of six spots found on three points which are located on the left and 3 right points are found. When more than three consecutive lines are found, it is considered only the first 3 thus will always have only one left lane on the control point $(\mathrm{PE})$ and only one control point in the right lane $(\mathrm{PD})$. Considering the variables shown in Figure 3(b) there have the coordinates $x$ and $y$ and the left lane of the control point (PE) are given by: 


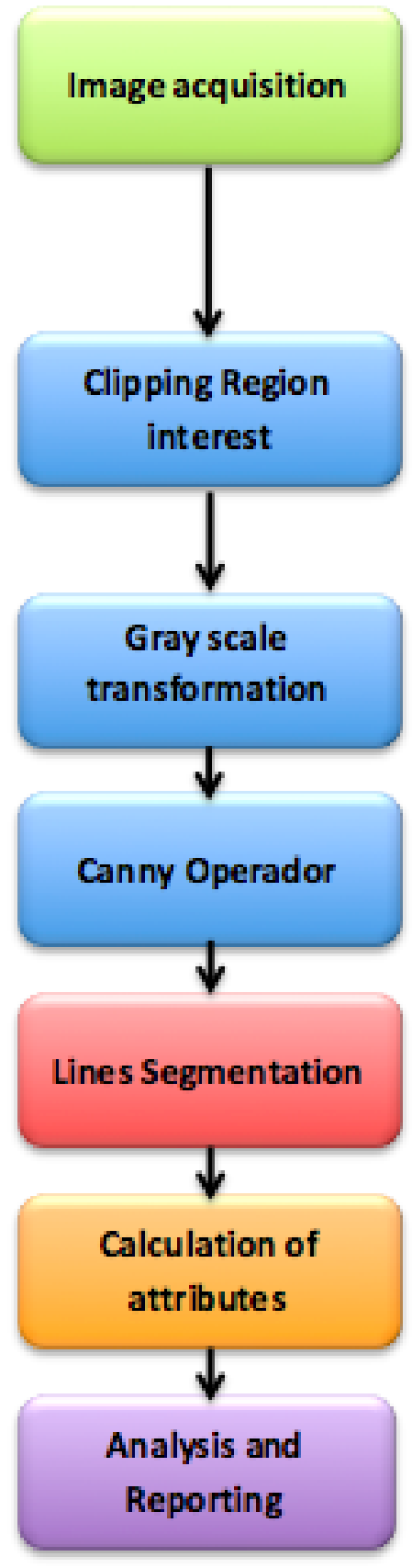

Fig. 2. Operating flow chart of the proposed system (ARCS).

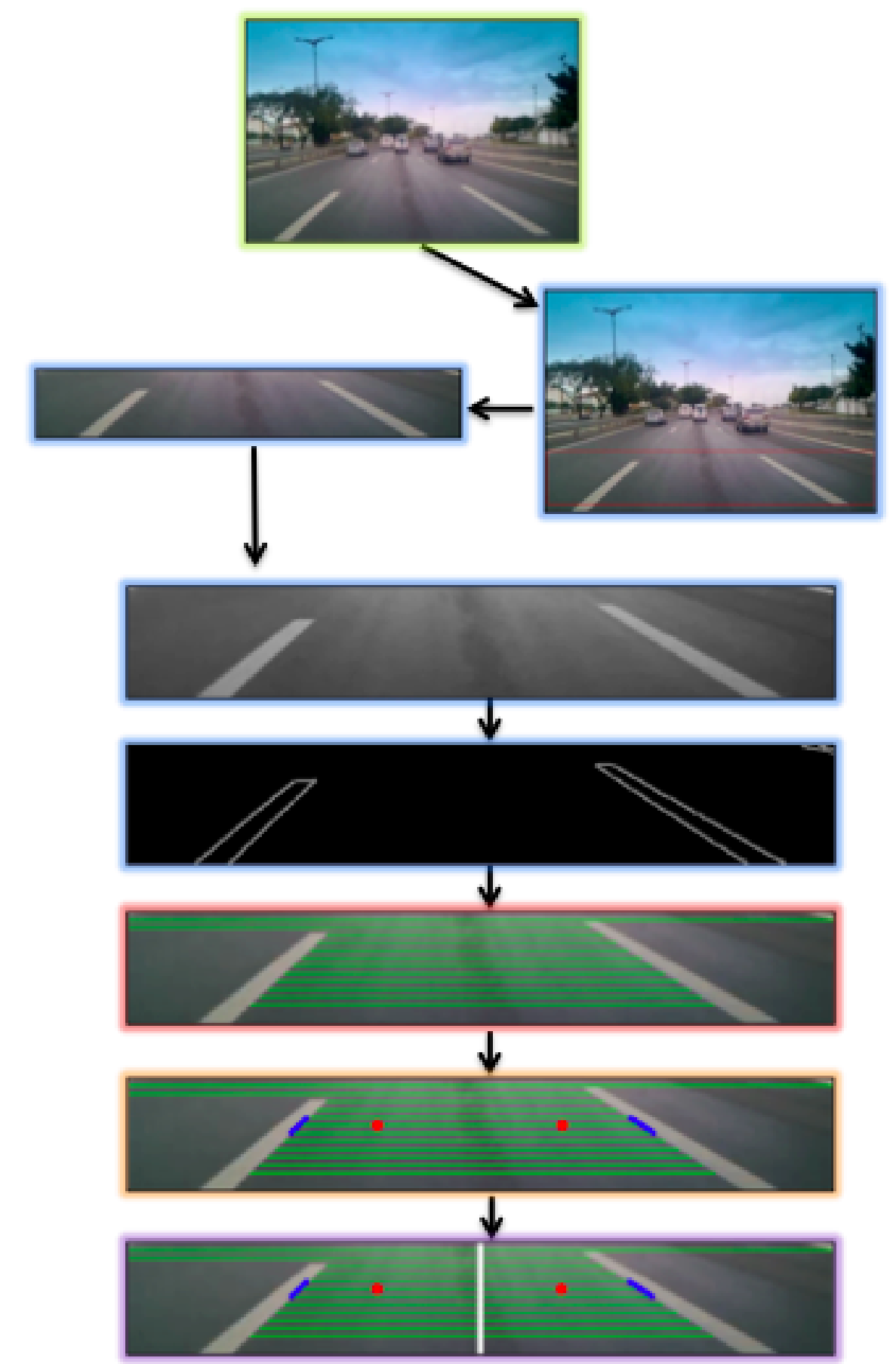

$$
x_{e}=\frac{x_{1}+x_{2}+x_{3}}{3}
$$

and

$$
y_{e}=\frac{y_{1}+y_{2}+y_{3}}{3}
$$

where $x_{d}$ and $y_{d}$ coordinates of the right lane of the control point (PD) is given by:

$$
x_{d}=\frac{x_{4}+x_{5}+x_{6}}{3}
$$




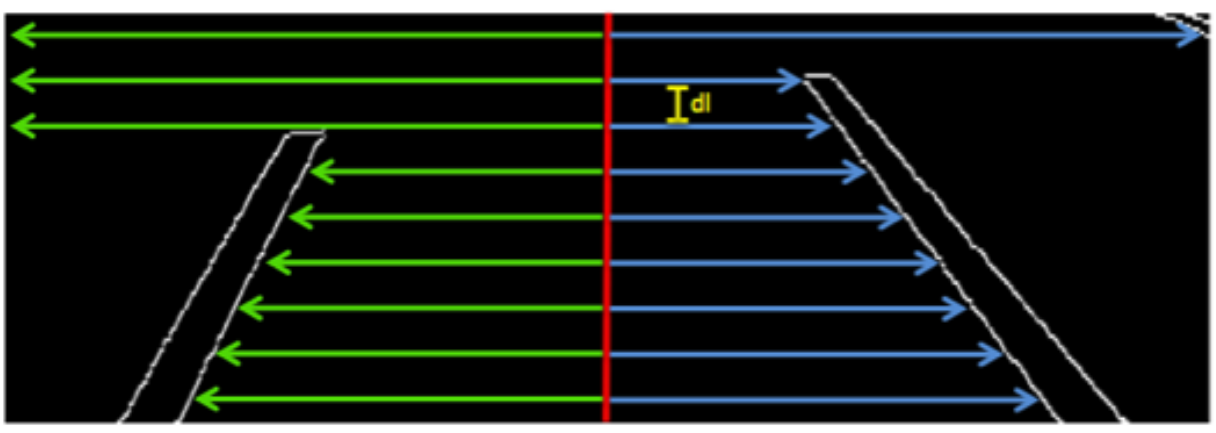

(a)

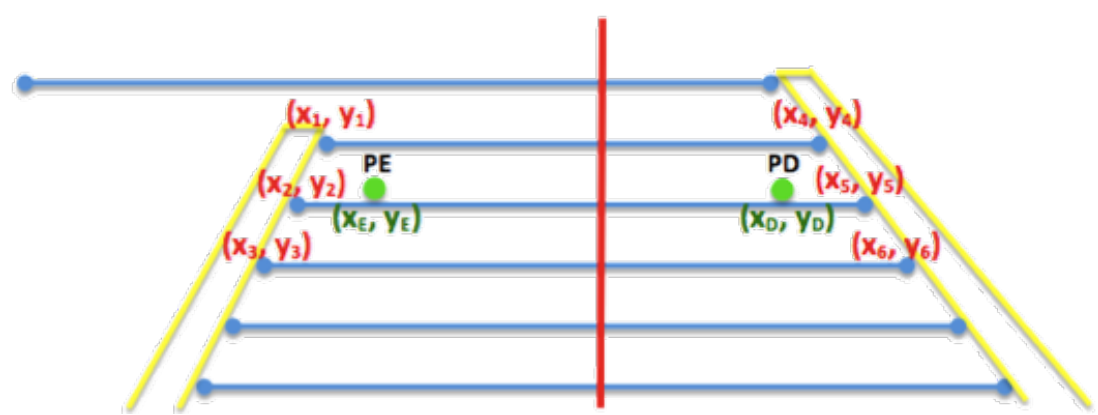

(b)

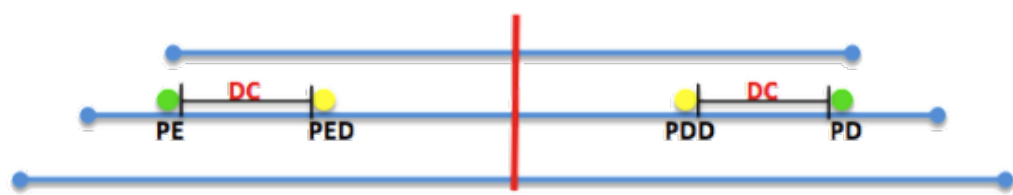

(c)

Fig. 3. Examples of detection methodology of vertical signaling tracks of the proposed system, and calculating the left of the control points (PE) and right (PD).

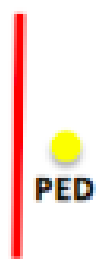

(a)

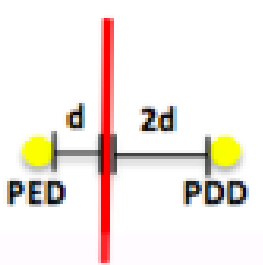

(b)

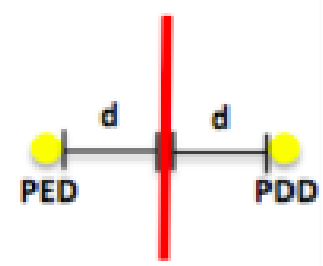

(c)

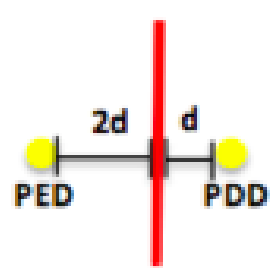

(d)

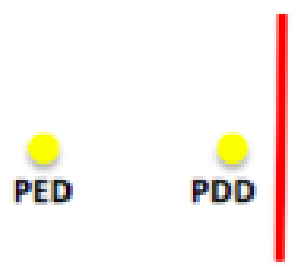

(e)

Fig. 4. Examples of analysis of the proposed system of the vehicle behavior.

and

$$
y_{d}=\frac{y_{4}+y_{5}+y_{6}}{3}
$$

After finding the $P E$ and $P D$ points, they are moved toward the center line of the image by a distance control (DC) configurable in the application generating the PED and PDD points, as shown in Figure 3(c)

\subsection{Analysis and Reporting}

The PED and PDD points found in the previous step are used at this stage to consider the location of the vehicle in relation to the found tracks. To do this analysis is used imaginary central line of the vehicle which represents the center thereof, as previously reported. The analysis generates this project has 5 possible outcomes relating the PED and PDD points with the center line of the image, where all these behaviors are illustrated in Figure 4 and listed below: 


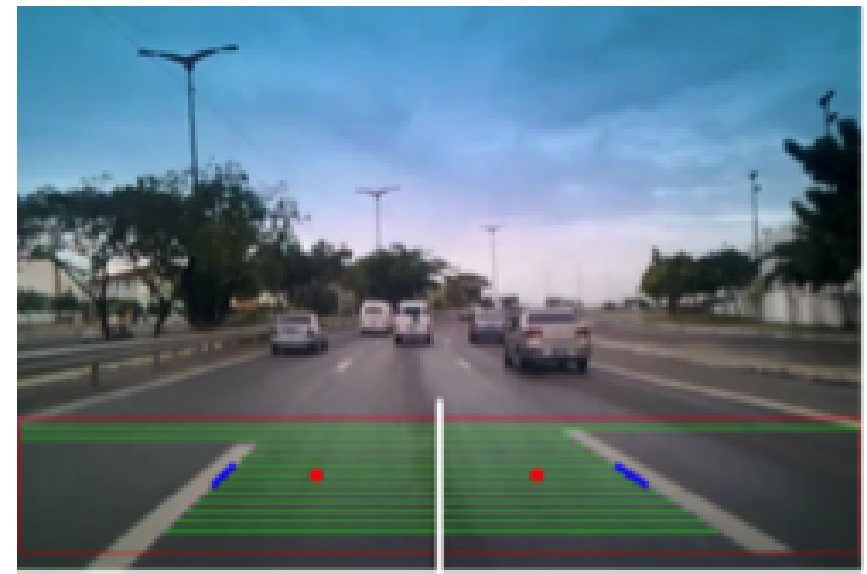

(a)

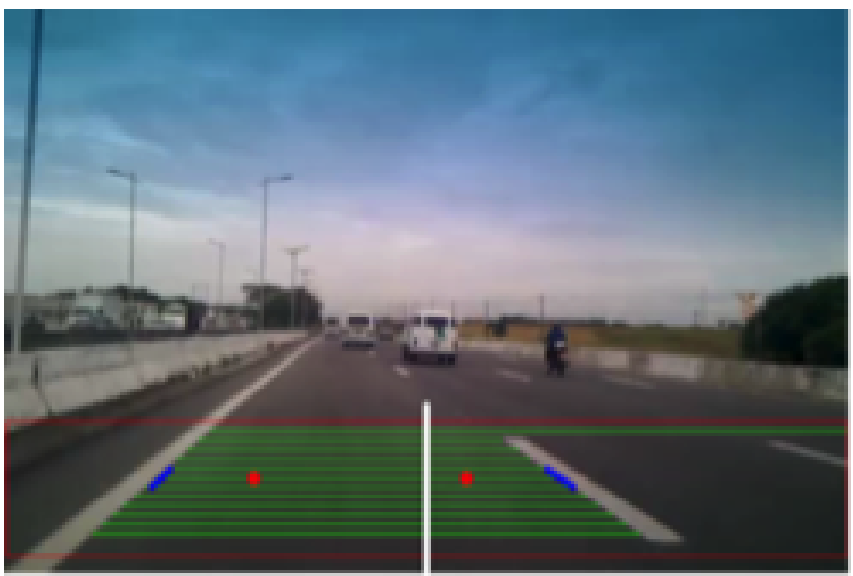

(c)

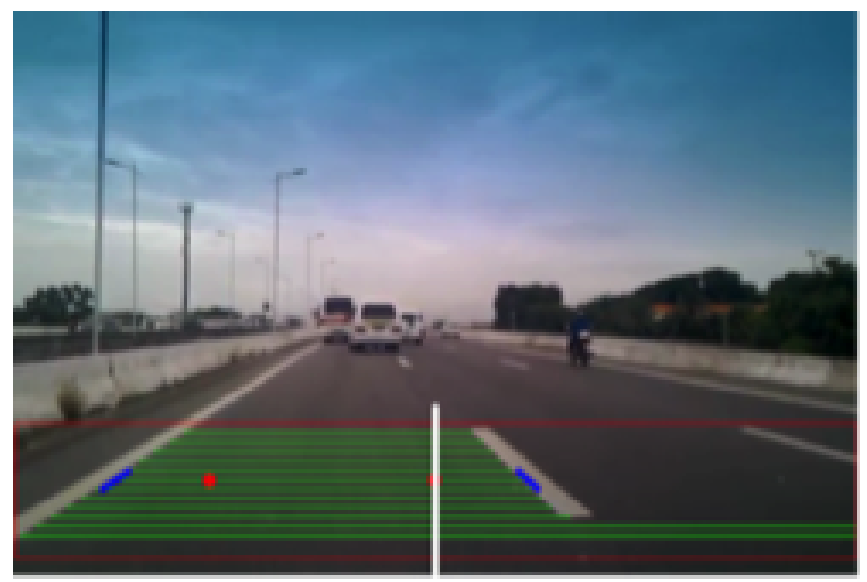

(e)

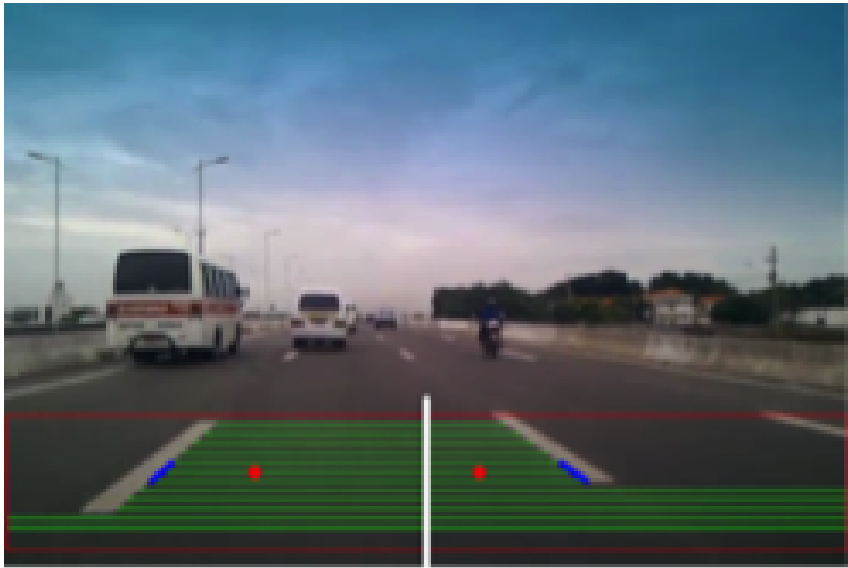

(b)

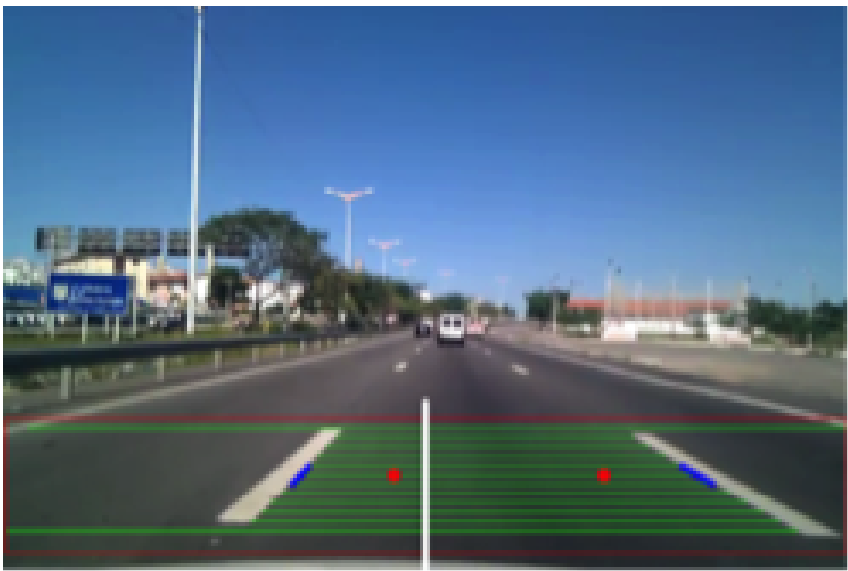

(d)

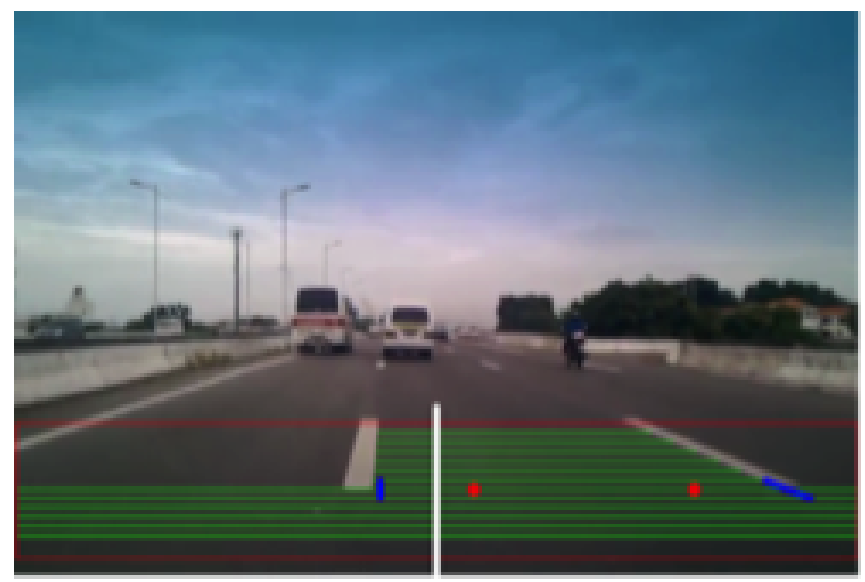

(f)

Fig. 5. Examples of results obtained by the proposed system with the analysis: a) centralized vehicle; b) and c) vehicle near the right track; d) vehicle near the left lane; e) the vehicle on the right-hand lane; f) the vehicle on the left lane.

(1) vehicle too close or on the left lane, when the center line is to the left of point EDP, as exemplified in Figure 4(a), 
(2) next vehicle left lane, when the distance from the PDD point for the central line is at least twice the distance of this line for developing countries, as exemplified in Figure 4(b)

(3) centered between the two strips vehicle, when the center line is between DCs and PDD with no distance is twice the other, as exemplified in Figure 4(c),

(4) Vehicle close to the right lane, when the distance from the PED point for the central line is at least twice the distance from this line to PDD, as exemplified in Figure 4(d).

(5) very close vehicle or on the right lane, when the center line is to the left of the PDD point, as exemplified in Figure 4(e) Two other behaviors are possible be detected when analyzing a sequence of two frames, which are described below:

(6) Change to the right lane, when in a previous frame the generated analysis was very close to the right lane (behavior 5) and the current frame analysis is very close to the left lane (1 behavior);

(7) change to the left lane: when in a previous frame the generated analysis was very close to the left lane (behavior 1) and in the current frame the analysis is very close to the right lane (behavior 5);

\section{EXPERIMENTAL TESTS AND DISCUSSIONS}

Tests conducted to validate the proposed system were performed in Unix platform on a computer I5 iMac with 8GB of RAM and HD 1TB getting the speed 50 frames per second (fps) and also on the Android platform on a Nexus device I getting 20fps. Performance tests were carried out on Brazilian highways, and some of the results can be seen in Figure 5 where three consecutive lines that detected the tracks are in blue, PED and PDD control of the points are in red and the line central are blank. It is noteworthy that the region considered for analysis is what is inside the red rectangle on the images shown in Figure 5

In the analysis of the outcomes of behavior analysis is computed similarity coefficient and sensitivity of these, since there are three possible types of results: True (T), False Positive (FP) and falsenegative (FN). The real case (V) is for the analyzes that had the actual behavior that the car was running; FP for behavior that the algorithm showed what was happening, but they were not; and behaviors that were not detected correspond to NFs. From the recording of these results, you can set the CS similarity coefficient and sensitivity $S$ in terms of percentage given by [7]:

$$
C S=\frac{2 V}{2 V+F N+F P} \times 100
$$

and

$$
S=\frac{V}{V+F N} \times 100
$$

Table 1 shows the results obtained by the proposed method for detecting behavior of motor vehicle drivers through horizontal traffic signs. The data present in this table consists of the number of occurrences for each type of detection sensitivity and coefficient of similarity.

Ao analisar a Tabela 1, percebe-se que a taxa geral dos coeficientes de similaridade e sensibilidade so, respectivamente, $97.27 \%$ e $97.47 \%$. Pode-se constatar tambm que todos os comportamentos analisados obtiveram ndices maiores que $90 \%$, demonstrando que o mtodo proposto promissor, possuindo resultados satisfatrios em todas as anlises.

In examining Table 1. one can see that the overall rate of similarity and sensitivity coefficients are, respectively, $97.27 \%$ and $97.47 \%$.
Table 1. Results obtained by the proposed method for analyzing driver behavior in vehicles.

\begin{tabular}{l|c|c|c|c|c}
\hline Behavior analysis & V & FN & FP & CS $(\%)$ & S $(\%)$ \\
\hline \hline 1. Very close vehicle track to the left & 47 & 1 & 2 & 96.90 & 97.91 \\
2. Vehicle near the track left & 45 & 2 & 3 & 94.73 & 95.74 \\
3. Centralized vehicle & 241 & 4 & 5 & 98.16 & 98.36 \\
4. Vehicle near the track right & 46 & 3 & 1 & 95.83 & 93.87 \\
5. Very close vehicle track to the right & 48 & 1 & 1 & 97.95 & 97.95 \\
6. Change to right lane & 19 & 0 & 1 & 97.43 & 100 \\
7. Change to left lane & 18 & 1 & 1 & 94.73 & 94.73 \\
\hline Total & 464 & 12 & 14 & 97.27 & 97.47 \\
\hline
\end{tabular}

Also can be seen that all behaviors analyzed had higher rates than $90 \%$, demonstrating that the proposed method is promising, having satisfactory results in all analyzes. Among the difficulties encountered by the proposed method in the motor vehicle behavior analysis using Digital Image Processing, the main is the detection of signal tracks, which in some highways virtually nonexistent.

Another difficulty is due to a few cars passing in front of the car and the analysis means that the car is a track, which even helps the driver, since the analysis will alert the driver should go in the other direction, contrary to car. However, this analysis is detected as a failure by the system and presented as False Positive in Table 1

\section{CONCLUSION AND FUTURE WORKS}

This paper proposes the Aid System the Driver Vehicle (ASDV), which is based on a system uses computer vision techniques and Digital Image Processing to detect the tracks of markings and through these identify the behavior that the car driver must have to continue in its tracks of movement on the road.

The tests show satisfactory results, achieving $100 \%$ accuracy when the tracks are detected.

It can be concluded that the system is effective in detecting the markings ranges and also in the analysis of the behavior of the vehicle, including generating alerts the driver assistance. The system showed good results on different platforms, demonstrating that has the potential to be used in commercial systems.

For future work, we intend to adapt the system to adverse conditions such as rain, nighttime, and perform the analysis to the detection of only one track. The intention is to use Artificial Intelligence techniques and Pattern Recognition to improve performance.

\section{Acknowledgements}

The authors acknowledge the financial support and encouragement from CNPq.

The authors thank the Post-Graduate Program in Renewable Energy from IFCE and Research Group in Intelligent Systems.

\section{REFERENCES}

[1] A. R. de Alexandria. System of optical recognition of digits to conventional energy meters. Master's thesis, Universidade Federal do Cear, Fortaleza, 2005.

[2] DNIT. Traffic accident statistics. national department of transport infrastructure. National Department of Transport Infrastructure of the Brazil, 2014.

[3] P. P. Rebouças Filho, R. M. Sarmento, Paulo Csar Cortez, A. C. S. BARROS, and VICTOR HUGO C. ALBUQUERQUE. Adaptive crisp active contour method for segmentation and reconstruction of $3 \mathrm{~d}$ lung structures. Revista Matria (UFRJ), 2015. 
[4] C. R. Franco. Identification of the use of seat belts in car drivers through computer vision. Master's thesis, UNIVALI, Itaja, 2013.

[5] S. L. Gomes, E. S. Rebouças, and P. P. Rebouças Filho. Reconhecimento optico de caracteres para reconhecimento das sinalizações verticais das vias de trânsito. Revista SODEBRAS, 9:9-12, 2014.

[6] R. C. Gonzalez and R.E. Woods. Digital Image Processing. Pearson Prentice Hall, New Jersey, 3a edition, 2010.

[7] J. N. Kaftan, A. P. Kiraly, A. Bakai, M. Das, C. L. Novak, and T. Aach. Fuzzy pulmonary vessel segmentation in contrast enhanced ct data. SPIE Proceedings, 2008.

[8] Takeo Kato, Yoshiki Ninomiya, and Ichiro Masaki. Preceding vehicle recognition based on learning from sample images. IEEE International Conference on Intelligent Transportation Systems, 3:252-260, 2002.

[9] H. Erdinc Kocer and K. Kursat Cevik. Artificial neural networks based vehicle license plate recognition. Procedia Computer Science, 3:1033-1037, 2011.

[10] Takashi Nato, Toshihiko Tsukada, Keiichi Yamada, Kazuhiro Kozuka, and Shin Yamamoto. Robust license-plate recognition methos for passing vehicles under outside environment. IEEE Transactions on Vehicular Technology, 49:2309-2319, 2000.

[11] E. Cavalcanti Neto, E. S. Rebouças, J. L. Moraes, S. L. Gomes, and P. P. Rebouças Filho. Development control parking access using techniques digital image processing and applied computational intelligence. IEEE Transactions on Latin America, 13:272-276, 2015.

[12] F. M. Peixoto, E. S. Rebouças, F. G. L. Xavier, and P. P. Rebouças Filho. Desenvolvimento de um software para clculo da densidade de ndulos de grafita em ferro fundido nodular atravs de processamento digital de imagens. Revista Matria (UFRJ), 2015.

[13] E. S. Rebouças, R. M. Sarmento, and P. P. Rebouças Filho. 3d adaptive balloon active contour: method of segmentation of structures in three dimensions. Revista IEEE Amrica Latina, 2015.

[14] Norizam Sulaiman, Sri Nor Hafidah Mohammad Jalani, Mahfuzah Mustafa, and Kamarul Hawari. Development of automatic vehicle plate detection system. In IEEE 3rd International Conference on System Engineering and Technology, 2013. 